\title{
Wave-Breaking Criterion for the Generalized Weakly Dissipative Periodic Two-Component Hunter-Saxton System
}

\author{
Jianmei Zhang and Lixin Tian \\ Department of Mathematics, Nonlinear Scientific Research Center, Jiangsu University, Zhenjiang, Jiangsu 212013, China \\ Correspondence should be addressed to Jianmei Zhang; jianmeizhang@sina.com
}

Received 22 May 2013; Accepted 22 July 2013

Academic Editor: Michael Meylan

Copyright (c) 2013 J. Zhang and L. Tian. This is an open access article distributed under the Creative Commons Attribution License, which permits unrestricted use, distribution, and reproduction in any medium, provided the original work is properly cited.

\begin{abstract}
This paper studies the wave-breaking criterion for the generalized weakly dissipative two-component Hunter-Saxton system in the periodic setting. We get local well-posedness for the generalized weakly dissipative two-component Hunter-Saxton system. We study a wave-breaking criterion for solutions and results of wave-breaking solutions with certain initial profiles.
\end{abstract}

\section{Introduction}

In recent years, the Hunter-Saxton equation [1]

$$
u_{t x x}+2 u_{x} u_{x x}+u u_{x x x}=0
$$

models the propagation of weakly nonlinear orientation waves in a massive nematic liquid crystal. In Hunter and Saxton [1], $x$ is the space variable in a reference frame moving with the linearized wave velocity, $t$ is a slow-time variable, and $u(t, x)$ is a measure of the average orientation of the medium locally around $x$ at time $t$. In order to be more precise, the orientation of the molecules is described by the field of unit vectors $(\cos u(t, x), \sin u(t, x))$ [2]. The HunterSaxton equation also arises in a different physical context as the high-frequency limit [3, 4] of the Camassa-Holm equation for shallow water waves $[5,6]$ and a reexpression of the geodesic flow on the diffeomorphism group of the circle [7] with a bi-Hamiltonian structure [1,8] which is completely integrable $[4,9]$. Hunter and Saxton [1] explored the initial value problem for the Hunter and Saxton equation on the line (nonperiodic case) and on the unit circle $S=$ $R / Z$ by using the method of characteristics, while Yin [2] studied it by using the Kato semigroup method. In addition, the two classes of admissible weak solutions, dissipative and conservative solutions, and their stability were investigated in [10-12]. Lenells [13] confirmed that the Hunter-Saxton equation also describes the geodesic flows on the quotient space of the infinite-dimensional group $D^{s}(S)$ modulo the subgroup of rotations $\operatorname{Rot}(S)$.

The Camassa-Holm equation admits many integrable multicomponent generalizations. So many authors studied the two-component Camassa-Holm system $[14,15]$. Inspired by this, recently, the researchers have made a study of the global existence of solutions to a two-component generalized Hunter-Saxton system in the periodic setting as follows:

$$
\begin{array}{r}
u_{t x x}+2 \sigma u_{x} u_{x x}+\sigma u u_{x x x}-\rho \rho_{x}+A u_{x}=0, \\
t>0, x \in R, \\
\rho_{t}+(\rho u)_{x}=0, \quad t>0, x \in R, \\
u(t, x+1)=u(t, x), \quad \rho(t, x+1)=\rho(t, x), \\
t \geq 0, x \in R, \\
u(0, x)=u_{0}(x), \quad \rho(0, x)=\rho_{0}(x), \quad x \in R .
\end{array}
$$

The authors of [16] have explored the particular choice of the parameter $\sigma=1$. The authors of [17] have further studied the wave breaking and global existence for the system for the parameter $\sigma \in \mathrm{R}$ to determine a wave-breaking criterion for strong solutions by using the localization analysis in the transport equation theory.

In general, avoiding energy dissipation mechanisms in a real world is not so easy. Wu and Yin $[18,19]$ have investigated the blow-up phenomena and the blow-up rate of the strong 
solutions of the weakly dissipative $\mathrm{CH}$ equation and DP equation. Inspired by the results mentioned above, we are going to discuss the initial value problem associated with the generalized weakly dissipative periodic two-component Hunter-Saxton system

$$
\begin{gathered}
u_{t x x}+2 \sigma u_{x} u_{x x}+\sigma u u_{x x x}-\rho \rho_{x}+A u_{x} \\
+\lambda\left(u-u_{x x}\right)=0, \quad t>0, x \in R, \\
\rho_{t}+(\rho u)_{x}=0, \quad t>0, x \in R, \\
u(t, x+1)=u(t, x), \quad \rho(t, x+1)=\rho(t, x), \\
t \geq 0, x \in R, \\
u(0, x)=u_{0}(x), \quad \rho(0, x)=\rho_{0}(x), \quad x \in R,
\end{gathered}
$$

where $\sigma \in R$ is the new free parameter and $A \geq 0, \lambda<0$.

Our major results of this paper are Theorems 11 and 12 (wave-breaking criterion). The remainder of the paper is organized as follows. Section 2 establishes the local wellposedness for (3) with the initial data in $H^{s} \times H^{s-1}, s \geq 2$. Section 3 deals with the wave breaking of this new system. Theorem 11, using transport equation theory, states a wavebreaking criterion which says that the wave breaking only depends on the slope of $u$, not the slope of $\rho$. Theorem 12 improves the blow-up criterion with a more precise condition.

Notation. Throughout this paper, $S=R / Z$ will denote the unit circle. By $H^{s}, s \geq 0$, we will represent the Sobolev spaces of equivalence classes of functions defined on the unit circle $S$ which have square-integrable distributional derivatives up to order $s$. The $H^{s}$-norm will be designated by $\|\cdot\|_{H^{s}}$, and the norm of a vector $v \in H^{s} \times H^{s-1}$ will be written as $\|v\|_{H^{s} \times H^{s-1}}$. Also, the Lebesgue spaces of order $p \in[1, \infty]$ will be denoted by $L^{p}(S)$, and the norm of their elements will be denoted by $\|f\|_{L^{p}(S)}$. Finally, if $p=2$, we agree on the convention $\|\cdot\|_{L^{2}(S)}=\|\cdot\|$.

\section{Preliminaries}

In this part, we will establish the local well-posedness for the Cauchy problem of system (3) by using Kato's theory. To pursue our goal, we give the results we wanted in brief.

We now provide the framework in which we will reformulate (3). To do this, we observe that we can write the first equation of (3) in the following integrated form:

$$
u_{t x}+\frac{\sigma}{2} u_{x}^{2}+\sigma u u_{x x}-\frac{1}{2} \rho^{2}+A u+\lambda \partial_{x}^{-1} u-\lambda u_{x}=g(t),
$$

where $\partial_{x}^{-1} f(x)=\int_{0}^{x} f(y) d y$ and $g(t)$ is determined by the periodicity of $u$ to be

$$
g(t)=-\int_{S}\left(\frac{\sigma}{2} u_{x}^{2}+\frac{1}{2} \rho^{2}-A u\right) d x
$$

Integrating both sides of (4) with respect to variable $x$, we get

$$
\begin{aligned}
u_{t}+\sigma u u_{x}= & \partial_{x}^{-1}\left(\frac{\sigma}{2} u_{x}^{2}+\frac{1}{2} \rho^{2}-A u+g+\lambda u_{x}-\lambda \partial_{x}^{-1} u\right) \\
& +h(t)
\end{aligned}
$$

where $h(t):[0, \infty) \rightarrow R$ is an arbitrary continuous function. Therefore, (3) can be written in the "transport" form as follows:

$$
\begin{gathered}
u_{t}+\sigma u u_{x}=\partial_{x}^{-1}\left(\frac{\sigma}{2} u_{x}^{2}+\frac{1}{2} \rho^{2}-A u-\lambda \partial_{x}^{-1} u+\lambda u_{x}+g\right) \\
+h(t), \quad t>0, x \in R, \\
\rho_{t}+u \rho_{x}=-u_{x} \rho, \quad t>0, x \in R, \\
u(t, x+1)=u(t, x), \quad \rho(t, x+1)=\rho(t, x), \\
t \geq 0, x \in R, \\
u(0, x)=u_{0}(x), \quad \rho(0, x)=\rho_{0}(x), \quad x \in R,
\end{gathered}
$$

where $h(t):[0, \infty) \rightarrow R$ is an arbitrary continuous function.

Next, we apply Kato's theory to establish the local wellposedness for the system (3). Consider the abstract quasilinear evolution equation

$$
\frac{d v}{d t}+A(v) v=f(v), \quad t \geq 0, v(0)=v_{0}
$$

Proposition 1 (see [20]). Given the evolution equation (8), assume that the Kato conditions hold. For a fixed $v_{0} \in Y$, there is a maximal $T>0$ depending only on $\left\|v_{0}\right\|_{Y}$ and a unique solution $v$ to the abstract quasi-linear evolution equation (8) such that

$$
v=v\left(\cdot v_{0}\right) \in C([0, T) ; Y) \cap C^{1}([0, T) ; X) .
$$

Moreover, the map $v_{0} \rightarrow v\left(\cdot, v_{0}\right)$ is continuous from $Y$ to

$$
C([0, T) ; Y) \cap C^{1}([0, T) ; X) .
$$

One may follow the similar argument as in [17] to obtain the following local well-posedness for (3).

Theorem 2. Given any $X_{0}=\left(\begin{array}{c}u_{0} \\ \rho_{0}\end{array}\right) \in H^{s} \times H^{s-1}, s \geq 2$, there exist a maximal $T=T\left(\sigma, A ;\left\|X_{0}\right\|_{H^{s} \times H^{s-1}}\right)>0$ and a unique solution $X=\left(\begin{array}{c}u \\ \rho\end{array}\right)$ to (3) such that

$$
\begin{aligned}
X=X\left(\cdot, X_{0}\right) & \in C\left([0, T) ; H^{s}(S) \times H^{s-1}(S)\right) \\
& \cap C^{1}\left([0, T) ; H^{s-1}(S) \times H^{s-2}(S)\right) .
\end{aligned}
$$

Moreover, the solution depends continuously on the initial data, that is, the mapping $X_{0} \rightarrow X\left(\cdot, X_{0}\right): H^{s} \times H^{s-1} \rightarrow$ $C\left([0, T) ; H^{s}(S) \times H^{s-1}(S)\right) \cap C^{1}\left([0, T)\right.$; and $H^{s-1}(S) \times$ $\left.H^{s-2}(S)\right)$ is continuous, and the maximal existence time $T$ can be chosen independently of the Sobolev orders. 
Now, discuss the initial value problem for the Lagrangian flow map as follows:

$$
\begin{gathered}
\frac{\partial \varphi}{\partial t}=u(t, \varphi(t, x)), \quad t \in[0, T), \\
\varphi(0, x)=x, \quad x \in R,
\end{gathered}
$$

where $u$ is the first component of the solution $X$ to (3). Using classical results from ordinary differential equations, one can acquire the following result on $\varphi$ which is of vital importance in the proof of the blow-up scenarios.

Lemma 3 (see [17]). Let $u \in C\left([0, T) ; H^{s}\right) \cap C^{1}\left([0, T) ; H^{s-1}\right)$, $s \geq 2$. Then, initial value problem (12) admits a unique solution $\varphi \in C^{1}([0, T) \times R, R)$. Moreover, $\{\varphi(t, \cdot)\}_{t \in[0, T)}$ is increasing diffeomorphism of $R$ with

$$
\varphi_{x}(t, x)=e^{\int_{0}^{T} u_{x}(\tau, \varphi(\tau, x)) d \tau}>0, \quad(t, x) \in[0, T) \times R .
$$

Remark 4. Since $\varphi(t, \cdot): R \rightarrow R$ is a diffeomorphism of the linear for every $t \in[0, T)$, the $L^{\infty}$-norm of any function $v(t, \cdot) \in L^{\infty}, t \in[0, T)$ is preserved under the family of diffeomorphisms $\varphi(t, \cdot)$ with $t \in[0, T)$, that is,

$$
\|v(t, \cdot)\|_{L^{\infty}(S)}=\|v(t, \varphi(t, \cdot))\|_{L^{\infty}(S)}, \quad t \in[0, T) .
$$

Similarly, we have

$$
\begin{array}{ll}
\inf _{x \in S} v(t, x)=\inf _{x \in S} v(t, \varphi(t, x)), & t \in[0, T), \\
\sup _{x \in S} v(t, x)=\sup _{x \in S} v(t, \varphi(t, x)), & t \in[0, T) .
\end{array}
$$

Lemma 5. Let $X_{0}=\left(\begin{array}{c}u_{0} \\ \rho_{0}\end{array}\right) \in H^{s} \times H^{s-1}, s \geq 2$, and let $T$ be the maximal existence time of the solution $X=\left(\begin{array}{c}u \\ \rho\end{array}\right)$ to (3) with initial data $X_{0}$. Then, for all $t \in[0, T)$, we have the following results:

$$
\begin{gathered}
\int_{S} \rho(t, x) d x=\int_{S} \rho_{0}(t) d x \\
\int_{S} u_{x}^{2}(t, x)+\rho^{2}(t, x) d x \leq \int_{S} u_{0, x}^{2}(x)+\rho_{0}^{2}(x) d x \triangleq E_{0} .
\end{gathered}
$$

Proof. On the one hand, integrating the second equation in (3) by parts and using the periodicity of $u$ and $\rho$, we acquire

$$
\frac{d}{d t} \int_{S} \rho d x=-\int_{S}(u \rho)_{x} d x=0 .
$$

On the other hand, multiplying (4) by $u_{x}$ and integrating by parts, considering the periodicity of $u$, we obtain

$$
\frac{d}{d t} \int_{S} u_{x}^{2} d x=-2 \int_{S} u \rho \rho_{x} d x+2 \lambda \int_{S} u^{2} d x+2 \lambda \int_{S} u_{x}^{2} d x
$$

Multiplying the second equation in (3) by $\rho$ and integrating by parts, we have

$$
\frac{d}{d t} \int_{S} \rho^{2} d x=2 \int_{S} u \rho \rho_{x} d x
$$

Adding the above two equations, we get

$$
\frac{d}{d t} \int_{S} u_{x}^{2}+\rho^{2} d x=2 \lambda\|u\|_{H^{2}}^{2}, \quad \lambda<0
$$

We acquire

$$
\int_{S} u_{x}^{2}(t, x)+\rho^{2}(t, x) d x \leq \int_{S} u_{0, x}^{2}(x)+\rho_{0}^{2}(x) d x \triangleq E_{0} .
$$

This completes the proof of Lemma 5.

Lemma 6. Let $X_{0}=\left(\begin{array}{c}u_{0} \\ \rho_{0}\end{array}\right) \in H^{s} \times H^{s-1}, s \geq 2$, and let $T$ be the maximal existence time of the solution $X=\left(\begin{array}{c}u \\ \rho\end{array}\right)$ to (3) with initial data $X_{0}$. Then, for all $t \in[0, T)$, we have the following results:

$$
\int_{S} u^{2}(t, x) d x \leq e^{C_{2} t}\left(\int_{S} u_{0}^{2}(x) d x+1\right),
$$

where $C_{1}=\max (|\sigma|-\lambda, 1) E_{0}+\sup _{t \in[0, \infty)}|h(t)|>0, C_{2}=$ $C_{1}+4 A-2 \lambda$.

Proof. By computing directly, we have

$$
\begin{aligned}
& \left|\partial_{x}^{-1}\left(\frac{\sigma}{2} u_{x}^{2}+\frac{1}{2} \rho^{2}-A u+g+\lambda u_{x}-\lambda \partial_{x}^{-1} u\right)+h(t)\right| \\
& \leq \int_{0}^{x}\left|\frac{\sigma}{2} u_{x}^{2}+\frac{1}{2} \rho^{2}-A u+g+\lambda u_{x}-\lambda \partial_{x}^{-1} u\right| d x+|h(t)| \\
& \leq \frac{1}{2} \max (|\sigma|-\lambda, 1) E_{0}+|g(t)|+|h(t)| \\
& \quad+(|A|-\lambda) \int_{0}^{1}|u| d x-\lambda \\
& \leq \max (|\sigma|-\lambda, 1) E_{0}+|h(t)|+(2|A|-\lambda) \int_{S}|u| d x-\lambda \\
& \quad:=C_{1}+(2|A|-\lambda) \int_{S}|u| d x-\lambda,
\end{aligned}
$$

where $C_{1}=\max (|\sigma|-\lambda, 1) E_{0}+\sup _{t \in[0, \infty)}|h(t)|>0$ and

$$
\begin{aligned}
|g(t)|= & \left|-\int_{S}\left(\frac{\sigma}{2} u_{x}^{2}+\frac{1}{2} \rho^{2}-A u\right) d x\right| \\
& \leq \frac{1}{2} \max (|\sigma|, 1) E_{0}+A \int_{S}|u| d x .
\end{aligned}
$$


Multiplying (6) by $u$ and integrating with respect to $x$, using the periodicity of $u$ and (24), we obtain

$$
\begin{aligned}
\frac{1}{2} \frac{d}{d t} \int_{S} u^{2}(t, x) d x \\
=\int_{S} u u_{t} d x \\
=-\sigma \int_{S} u_{x} u^{2} d x \\
\quad+\int_{S} u\left[\partial _ { x } ^ { - 1 } \left(\frac{\sigma}{2} u_{x}^{2}+\frac{1}{2} \rho^{2}-A u+\lambda u_{x}\right.\right. \\
=\int_{S} u\left[\partial _ { x } ^ { - 1 } \left(\frac{\sigma}{2} u_{x}^{2}+\frac{1}{2} \rho^{2}-A u+\lambda u_{x}\right.\right. \\
\leq \quad\left(C_{1}+(2|A|-\lambda) \int_{S}|u| d x-\lambda\right) \int_{S}|u| d x \\
\left.\left.\leq \quad \frac{C_{2}}{2} \int_{S} u^{2} d x+\frac{C_{1}-\lambda}{2}+(2|A|-2 \lambda)\right) \int_{S}^{-1} u+g\right)+h(t) u^{2} d x+\frac{C_{1}-\lambda}{2} \\
\leq C_{S}|u| d x+(2|A|-\lambda)\left(\int_{S}|u| d x\right)^{2}-\lambda \int_{S} u^{2} d x
\end{aligned}
$$

where $C_{2}=C_{1}+4 A-2 \lambda$; note that $C_{2}>C_{1}$.

By Gronwall's inequality, we get

$$
\begin{aligned}
\int_{S} u^{2}(t, x) d x & \leq e^{C_{2} t}\left(\int_{S} u_{0}^{2}(x) d x+\frac{C_{1}-\lambda}{C_{2}}\right)-\frac{C_{1}-\lambda}{C_{2}} \\
& \leq e^{C_{2} t}\left(\int_{S} u_{0}^{2}(x) d x+1\right) .
\end{aligned}
$$

This completes the proof of Lemma 6.

Lemma 7. Assume that $u_{0} \in H^{s}(S), s \geq 2, u_{0} \neq 0$, and that the corresponding solution $u(t, x)$ of (3) has a zero point for any time $t \geq 0$. Then, for all $t \in[0, T)$ we have

$$
\int_{S} u^{2}(t, x) d x \leq \int_{S} u_{x}^{2}(t, x) d x \leq E_{0} .
$$

Proof. By assumption, there is $x_{0} \in[0,1]$ such that $u\left(t, x_{0}\right)=$ 0 for each $t \in[0, T)$.

Then, for $x \in S$, by holder equality, we have

$$
\begin{array}{r}
u^{2}(t, x)=\left(\int_{x_{0}}^{x} u_{x} d x\right)^{2} \leq \\
x \in\left[x_{0}, x_{0}+\frac{1}{2}\right] .
\end{array}
$$

This implies $\sup _{x \in S} u^{2}(t, x) \leq(1 / 2) \int_{S} u_{x}^{2} d x$

$$
\begin{aligned}
\int_{S} u^{2}(t, x) d x & \leq \sup _{x \in S} u^{2}(t, x) \leq \frac{1}{2} \int_{S} u_{x}^{2} d x \\
& \leq \int_{S} u_{x}^{2}+\rho^{2} d x \leq E_{0}
\end{aligned}
$$

\section{Wave-Breaking Criteria}

In this section, by using transport equation theory, we obtain the wave-breaking criteria for solutions to (3). We first recall the following propositions.

Proposition 8 (1D Moser-type estimates). The following estimates hold:

(a) For $s \geq 0$,

$\|f g\|_{H^{s}(R)} \leq C\left(\|f\|_{L^{\infty}(R)}\|g\|_{H^{s}(R)}+\|f\|_{H^{s}(R)}\|g\|_{L^{\infty}(R)}\right)$.

(b) For $s>0$,

$\left\|f \partial_{x} g\right\|_{H^{s}(R)} \leq C\left(\|f\|_{L^{\infty}(R)}\left\|\partial_{x} g\right\|_{H^{s}(R)}+\|f\|_{H^{s+1}(R)}\|g\|_{L^{\infty}(R)}\right)$.

(c) For $s_{1} \leq 1 / 2, s_{2}>1 / 2, s_{1}+s_{2}>0$,

$$
\|f g\|_{H^{s_{1}(R)}} \leq C\|f\|_{H^{s_{1}(R)}}\|g\|_{H^{s_{2}(R)}},
$$

where $C^{\prime}$ s are constants that are independent of $f$ and $g$.

Proposition 9 (see [21]). Suppose that $s>-d / 2$. Let $v$ be a vector field such that $\nabla v$ belongs to $L^{1}\left([0, T] ; H^{s-1}\right)$ if $s>1+d / 2$ or to $L^{1}\left([0, T] ; H^{d / 2} \cap L^{\infty}\right)$, otherwise. Suppose also that $f_{0} \in H^{s}, F \in L^{1}\left([0, T] ; H^{s}\right)$ and that $f \in$ $L^{\infty}\left([0, T] ; H^{s}\right) \cap C\left([0, T] ; S^{\prime}\right)$ solves the d-dimensional linear transport equations

$$
(T)\left\{\begin{array}{l}
\partial_{t} f+v \cdot \nabla f=F \\
\left.f\right|_{t=0}=f_{0}
\end{array}\right.
$$

Then $f \in C\left([0, T] ; H^{s}\right)$. More precisely, there exists a constant $C$ depending only on $s, p$, and $d$ such that the following statements hold:

(1) If $s \neq 1+d / 2$,

$\|f\|_{H^{s}} \leq\left\|f_{0}\right\|_{H^{s}}+\int_{0}^{t}\|F(\tau)\|_{H^{s}} d \tau+C \int_{0}^{t} V^{\prime}(\tau)\|f(\tau)\|_{H^{s}} d \tau$,

or

$$
\|f\|_{H^{s}} \leq e^{C V(t)}\left(\left\|f_{0}\right\|_{H^{s}}+\int_{0}^{t} e^{-C V(\tau)}\|F(\tau)\|_{H^{s}} d \tau\right),
$$

with $V(t)=\int_{0}^{t}\|\nabla v(\tau)\|_{H^{d / 2} \cap L^{\infty}} d \tau$ if $s<1+d / 2$ and $V(t)=$ $\int_{0}^{t}\|\nabla v(\tau)\|_{H^{s-1}} d \tau$ else. 
(2) If $f=v$, then for all $s>0$, estimates (35) and (36) hold with

$$
V(t)=\int_{0}^{t}\left\|\partial_{x} u(\tau)\right\|_{L^{\infty}} d \tau
$$

Proposition 10 (see [21]). Let $0<s<1$. Suppose that $f_{0} \in$ $H^{s}, g \in L^{1}\left([0, T] ; H^{s}\right) v, \partial_{x} v \in L^{1}\left([0, T] ; L^{\infty}\right)$ and that $f \in$ $L^{\infty}\left([0, T] ; H^{s}\right) \cap C\left([0, T] ; S^{\prime}\right)$ solves the 1-dimensional linear transport equation

$$
\text { (T) }\left\{\begin{array}{l}
\partial_{t} f+v \cdot \nabla f=g \\
\left.f\right|_{t=0}=f_{0}
\end{array}\right.
$$

Then $f \in C\left([0, T] ; H^{s}\right)$. More precisely, there exists a constant $C$ depending only on s such that the following statements hold:

$$
\begin{aligned}
\|f\|_{H^{s}} \leq\left\|f_{0}\right\|_{H^{s}} & +C \int_{0}^{t}\|g(\tau)\|_{H^{s}} d \tau \\
& +C \int_{0}^{t} V^{\prime}(\tau)\|f(\tau)\|_{H^{s}} d \tau
\end{aligned}
$$

or

$$
\|f\|_{H^{s}} \leq e^{C V(t)}\left(\left\|f_{0}\right\|_{H^{s}}+C \int_{0}^{t}\|g(\tau)\|_{H^{s}} d \tau\right)
$$

with $V(t)=\int_{0}^{t}\left(\|v(\tau)\|_{L^{\infty}}+\left\|\partial_{x} v(\tau)\right\|_{L^{\infty}}\right) d \tau$.

The above proposition was proved in [8] using Littlewood-Paley analysis for the transport equation and Mosertype estimates. Using this result and performing the same argument, as in [17], we can obtain the following blow-up criterion.

Theorem 11. Let $X_{0}=\left(\begin{array}{c}u_{0} \\ \rho_{0}\end{array}\right) \in H^{s} \times H^{s-1}$ with $s \geq 2$, and $X=$ $\left(\begin{array}{l}u \\ \rho\end{array}\right)$ be the corresponding solution to (3). Assume that $T>0$ is the maximal time of existence. Then

$$
T<\infty \Longrightarrow \int_{0}^{T}\left\|\partial_{x} u(\tau)\right\|_{L^{\infty}} d \tau=\infty
$$

Our next result describes the necessary and sufficient condition for the blow-up of solutions to (3).

Theorem 12. Suppose that $\sigma \in R \backslash\{0\}$. Let $X_{0}=\left(\begin{array}{c}u_{0} \\ \rho_{0}\end{array}\right) \in H^{s} \times$ $H^{s-1}$, with $s \geq 2$, and let $T$ be the maximal existence time of the solution $X=\left(\begin{array}{c}u \\ \rho\end{array}\right)$ to (3) with initial data $X_{0}$. Then, the solution blows up in finite time if and only if

$$
\liminf _{t \rightarrow T^{-}}\left\{\inf _{x \in S} \sigma u_{x}(t, x)\right\}=-\infty .
$$

The approach one takes here is the method of characteristics. Applying the following lemma, we may carry out the estimates along the characteristics $\varphi(t, x)$ which captures $\sup _{x \in S} u_{x}(t, x)$ and $\inf _{x \in S} u_{x}(t, x)$.
Lemma 13 (see [22]). Let $T>0$ and let $v \in C^{1}\left([0, T] ; H^{2}(R)\right)$. Then, for every $t \in[0, T)$, there exists at least one point $\xi(t) \in R$ with

$$
m(t):=\inf _{x \in S} v_{x}(t, x)=v_{x}(t, \xi(t)),
$$

and the function $m(t)$ is almost everywhere differentiable on $(0, T)$ with

$$
\frac{d m(t)}{d t}=v_{t x}(t, \xi(t)), \quad \text { a.e.on }(0, T) .
$$

Lemma 14. Let $X_{0}=\left(\begin{array}{c}u_{0} \\ \rho_{0}\end{array}\right) \in H^{s} \times H^{s-1}$ with $s \geq 2$, and let $T$ be the maximal existence time of the solution $X=\left(\begin{array}{c}u \\ \rho\end{array}\right)$ to (3) with initial data $X_{0}$. Then one has the following:

(1) $\sigma \neq 0$

$$
\begin{aligned}
\sup _{x \in S} u_{x}(t, x) \leq & \left\|u_{0, x}\right\|_{L^{\infty}(S)} \\
& +\sqrt{\frac{\left\|\rho_{0}\right\|_{L^{\infty}(S)}^{2}+K_{1}^{2}(T)}{\sigma}+\frac{\lambda^{2}}{\sigma^{2}}}+\frac{\lambda}{\sigma}
\end{aligned}
$$

$(\sigma>0)$

$$
\begin{aligned}
\inf _{x \in S} u_{x}(t, x) \geq & -\left\|u_{0, x}\right\|_{L^{\infty}(S)} \\
& -\sqrt{\frac{\lambda^{2}}{\sigma^{2}}-\frac{K_{2}^{2}(T)}{\sigma}}+\frac{\lambda}{\sigma} \quad(\sigma<0) .
\end{aligned}
$$

(2) $\sigma=0$

$$
\begin{aligned}
& \sup _{x \in S} u_{x}(t, x) \leq \sup _{x \in S} u_{0, x}(x) \\
&+\frac{1}{2}\left(\sup _{x \in S} \rho_{0}^{2}(x)+K_{1}^{2}(T)\right) \frac{e^{\lambda t}-1}{\lambda} \\
& \inf _{x \in S} u_{x}(t, x) \geq \inf _{x \in S} u_{0, x}(x) \\
&+\frac{1}{2}\left(\inf _{x \in S} \rho_{0}^{2}(x)-K_{2}^{2}(T)\right) \frac{e^{\lambda t}-1}{\lambda} .
\end{aligned}
$$

The constants above are defined as follows:

$$
\begin{aligned}
& K_{1}(T)=\sqrt{2 A-\lambda+\frac{A}{2} E_{0}+\frac{3 A-2 \lambda}{2}\left[e^{C_{2} T}\left(\left\|u_{0}\right\|_{L^{2}(S)}^{2}+1\right)\right]}, \\
& K_{2}(T) \\
& =\sqrt{2 A-\lambda+\frac{A+2}{2} E_{0}+\frac{3 A-2 \lambda}{2}\left[e^{C_{2} T}\left(\left\|u_{0}\right\|_{L^{2}(S)}^{2}+1\right)\right] .}
\end{aligned}
$$

Proof of Lemma 14. By Theorem 2 and a simple density argument, we show that the desired results are valid when $s \geq 3$, so we take $s=3$ in the proof. 
Let $\sigma>0$. Using Lemma 13 and the fact that

$$
\sup _{x \in S}\left[v_{x}(t, x)\right]=-\inf _{x \in S}\left[-v_{x}(t, x)\right]
$$

We can consider $M(t)$ and $\gamma(t)$ as follows:

$$
M(t):=u_{x}(t, \xi(t))=\sup _{x \in S}\left[u_{x}(t, x)\right], \quad t \in[0, T) .
$$

Hence,

$$
u_{x x}(t, \xi(t))=0, \quad \text { a.e. on } t \in[0, T)
$$

Take the trajectory $\varphi(t, x)$ defined in (12). Then we know that $\varphi(t, \cdot): R \rightarrow R$ is a diffeomorphism for every $t \in[0, T)$. Therefore, there exists $x_{0}(t) \in R$ such that

$$
\varphi\left(t, x_{0}(t)\right)=\xi(t), \quad t \in[0, T)
$$

Now, let

$$
\gamma(t)=\rho\left(t, \varphi\left(t, x_{0}\right)\right), \quad t \in[0, T)
$$

Therefore, along the trajectory $\varphi\left(t, x_{0}\right),(4)$ and the second equation of (3) become

$$
\begin{gathered}
M^{\prime}(t)=-\frac{\sigma}{2} M^{2}(t)+\lambda M(t)+\frac{1}{2} \gamma^{2}(t)+f\left(t, \varphi\left(t, x_{0}\right)\right) \\
\gamma^{\prime}(t)=-\gamma M, \quad \text { a.e. } t \in[0, T),
\end{gathered}
$$

where the notation denotes the derivative with respect to $t$ and $f$ represents the function

$$
\begin{aligned}
f & =-A u-\lambda \partial_{x}^{-1} u+g(t) \\
& =-A u-\lambda \partial_{x}^{-1} u-\int_{S}\left(\frac{\sigma}{2} u_{x}^{2}+\frac{1}{2} \rho^{2}-A u\right) d x
\end{aligned}
$$

We first compute the upper and lower bounds for $f$ for later use in getting the blow-up result as follows:

$$
\begin{aligned}
f= & -A u-\lambda \int_{0}^{x} u d x-\frac{\sigma}{2} \int_{S} u_{x}^{2} d x-\frac{1}{2} \int_{S} \rho^{2} d x+A \int_{S} u d x \\
\leq & -A u-\lambda \int_{0}^{x} u d x+A \int_{S} u d x \leq \frac{A}{2}\left(1+u^{2}\right) \\
& +\frac{A-\lambda}{2}\left(1+\int_{S} u^{2} d x\right) .
\end{aligned}
$$

Since $u^{2} \leq(1 / 2) \int_{S}\left(u^{2}+u_{x}^{2}\right) d x,(17)$, we obtain the upper bound for $f$

$$
\begin{aligned}
f \leq & \frac{A}{2}\left(1+\frac{1}{2} \int_{S}\left(u^{2}+u_{x}^{2}\right) d x\right)+\frac{A-\lambda}{2}\left(1+\int_{S} u^{2} d x\right) \\
\leq & A-\frac{\lambda}{2}+\frac{A}{4} \int_{S}\left(\rho^{2}+u_{x}^{2}\right) d x+\frac{3 A-2 \lambda}{4} \int_{S} u^{2} d x \\
\leq & A-\frac{\lambda}{2}+\frac{A}{4} E_{0}+\frac{3 A-2 \lambda}{4}\left[e^{C_{2} t}\left(\int_{S} u_{0}^{2}(x) d x+1\right)\right] \\
\leq & A-\frac{\lambda}{2}+\frac{A}{4} E_{0}+\frac{3 A-2 \lambda}{4} \\
& \times\left[e^{C_{2} T}\left(\left\|u_{0}\right\|_{L^{2}(S)}^{2}+1\right)\right]:=\frac{1}{2} K_{1}^{2}(T) .
\end{aligned}
$$

Now we turn to the lower bound of $f$. Using previous arguments, we get

$$
\begin{aligned}
-f= & A u+\lambda \int_{0}^{x} u d x+\frac{\sigma}{2} \int_{S} u_{x}^{2} d x+\frac{1}{2} \int_{S} \rho^{2} d x-A \int_{S} u d x \\
\leq & A \frac{1+u^{2}}{2}+\frac{\max (|\sigma|, 1)}{2} \int_{S}\left(\rho^{2}+u_{x}^{2}\right) d x \\
& +\frac{A-\lambda}{2}\left(1+\int_{S} u^{2} d x\right) \\
\leq & A-\frac{\lambda}{2}+\frac{A+2 \max (|\sigma|, 1)}{4} \int_{S}\left(\rho^{2}+u_{x}^{2}\right) d x \\
& +\frac{3 A-2 \lambda}{4} \int_{S} u^{2} d x \\
\leq & A-\frac{\lambda}{2}+\frac{A+2 \max (|\sigma|, 1)}{4} E_{0} \\
& +\frac{3 A-2 \lambda}{4}\left[e^{C_{2} T}\left(\left\|u_{0}\right\|_{L^{2}(S)}^{2}+1\right)\right] .
\end{aligned}
$$

When $\sigma<0$, we have a finer estimate

$$
\begin{aligned}
-f \leq & A-\frac{\lambda}{2}+\frac{A+2}{4} \int_{S}\left(\rho^{2}+u_{x}^{2}\right) d x+\frac{3 A-2 \lambda}{4} \int_{S} u^{2} d x \\
\leq & A-\frac{\lambda}{2}+\frac{A+2}{4} E_{0}+\frac{3 A-2 \lambda}{4} \\
& \times\left[e^{C_{2} T}\left(\left\|u_{0}\right\|_{L^{2}(S)}^{2}+1\right)\right]:=\frac{1}{2} K_{2}^{2}(T) .
\end{aligned}
$$

Combining (59) and (60), we obtain

$$
\begin{aligned}
|f| \leq & A-\frac{\lambda}{2}+\frac{A+2 \max (|\sigma|, 1)}{4} E_{0} \\
& +\frac{3 A-2 \lambda}{4}\left[e^{C_{2} T}\left(\left\|u_{0}\right\|_{L^{2}(S)}^{2}+1\right)\right]:=\frac{1}{2} K_{3}^{2}(T) .
\end{aligned}
$$

Since $s \geq 3$, we have $u \in C_{0}^{1}(S)$. Therefore,

$\sup _{x \in S} u_{x}(t, x) \geq 0, \quad \inf _{x \in S} u_{x}(t, x) \leq 0, \quad t \in[0, T)$. 
Hence, $M(t)>0$ for $t \in[0, T)$. From the second equation of (55), we obtain

$$
\gamma(t)=\gamma(0) e^{-\int_{0}^{t} M(\tau) d \tau}
$$

Hence,

$$
\left|\rho\left(t, \varphi\left(t, x_{0}\right)\right)\right|=|\gamma(t)| \leq|\gamma(0)| \leq\left\|\rho_{0}\right\|_{L^{\infty}(S)} .
$$

For any given $x \in S$, define

$$
\begin{aligned}
p_{1}(t)= & M(t)-\left\|u_{0, x}\right\|_{L^{\infty}(S)} \\
& -\sqrt{\frac{\left\|\rho_{0}\right\|_{L^{\infty}(S)}^{2}+K_{1}^{2}(T)}{\sigma}+\frac{\lambda^{2}}{\sigma^{2}}}-\frac{\lambda}{\sigma} \quad(\sigma>0) .
\end{aligned}
$$

Observing that $p_{1}(t)$ is a $C^{1}$-differentiable function on $[0, T)$ and satisfies

$$
\begin{aligned}
p_{1}(0)= & M(0)-\left\|u_{0, x}\right\|_{L^{\infty}(S)} \\
& -\sqrt{\frac{\left\|\rho_{0}\right\|_{L^{\infty}(S)}^{2}+K_{1}^{2}(T)}{\sigma}+\frac{\lambda^{2}}{\sigma^{2}}}-\frac{\lambda}{\sigma} \\
\leq & M(0)-\left\|u_{0, x}\right\|_{L^{\infty}(S)} .
\end{aligned}
$$

We now claim that $p_{1}(t) \leq 0 t \in[0, T)$.

Assume the contrary that there is $t_{0} \in[0, T)$ such that $p_{1}\left(t_{0}\right)>0$

Let $t_{1}=\max \left\{t<t_{0}: p_{1}(t)=0\right\}$. Then $p_{1}\left(t_{1}\right)=0$ and $p_{1}^{\prime}\left(t_{1}\right) \geq 0$, or equivalently,

$$
M\left(t_{1}\right)=\left\|u_{0, x}\right\|_{L^{\infty}(S)}+\sqrt{\frac{\left\|\rho_{0}\right\|_{L^{\infty}(S)}^{2}+K_{1}^{2}(T)}{\sigma}+\frac{\lambda^{2}}{\sigma^{2}}}+\frac{\lambda}{\sigma}
$$

and $M^{\prime}\left(t_{1}\right) \geq 0$ a.e. $t \in[0, T)$. On the other hand, we have

$$
\begin{aligned}
M^{\prime}\left(t_{1}\right)= & -\frac{\sigma}{2} M^{2}\left(t_{1}\right)+\lambda M\left(t_{1}\right)+\frac{1}{2} \gamma^{2}\left(t_{1}\right) \\
& +f\left(t_{1}, \varphi\left(t_{1}, x_{0}\right)\right) \quad \text { a.e. } t \in[0, T) \\
\leq & -\frac{\sigma}{2}\left(M\left(t_{1}\right)-\frac{\lambda}{\sigma}\right)^{2}+\frac{1}{2}\left\|\rho_{0}\right\|_{L^{\infty}(S)}^{2}+\frac{\lambda^{2}}{2 \sigma} \\
& +\frac{K_{1}^{2}(T)}{2}<0,
\end{aligned}
$$

which is a contradiction. Therefore, $p_{1}(t) \leq 0$ for all $t \in[0, T)$. Since $x$ is arbitrarily chosen, we obtain (45).

To derive (46) in the case of $\sigma<0$, we consider $\widetilde{M}(t)$ and $\widetilde{\gamma}(t)$ as in Lemma 13:

$$
\widetilde{M}(t):=u_{x}(t, \zeta(t))=\inf _{x \in S}\left[u_{x}(t, x)\right] \quad t \in[0, T)
$$

Hence,

$$
u_{x x}(t, \zeta(t))=0 \quad \text { a.e. } t \in[0, T) .
$$

Using previous arguments, we take the characteristic $\varphi(t$, $x$ ) defined in (13) and choose $x_{1}(t) \in R$ such that

$$
\varphi\left(t, x_{1}(t)\right)=\zeta(t)
$$

Let

$$
\widetilde{\gamma}(t)=\rho(t, \varphi(t, x)), \quad t \in[0, T)
$$

Hence, along the trajectory $\widetilde{M}^{\prime}(t)=\lambda \widetilde{M}(t)+(1 / 2) \gamma^{2}(t)+$ $f\left(t, \varphi\left(t, x_{0}\right)\right) \geq \lambda \widetilde{M}(0)+(1 / 2) \gamma^{2}(0)+(1 / 2) K_{2}^{2}(T),(4)$ and the second equation of (3) become

$$
\begin{gathered}
\widetilde{M}^{\prime}(t)=-\frac{\sigma}{2} \widetilde{M}^{2}(t)+\lambda \widetilde{M}(t)+\frac{1}{2} \gamma^{2}(t)+f\left(t, \varphi\left(t, x_{0}\right)\right) \\
\widetilde{\gamma}^{\prime}(t)=-\widetilde{\gamma} \widetilde{M}, \quad \text { a.e. } t \in[0, T) .
\end{gathered}
$$

Define

$$
\begin{aligned}
p_{2}(t)= & \widetilde{M}(t)+\left\|u_{0, x}\right\|_{L^{\infty}(S)} \\
& +\sqrt{\frac{\lambda^{2}}{\sigma^{2}}-\frac{K_{2}^{2}(T)}{\sigma}-\frac{\lambda}{\sigma}} \quad(\sigma<0) .
\end{aligned}
$$

For any given $x \in S$, Note that $p_{2}(t)$ is also $C^{1}$-differentiable function on $[0, T)$ and satisfies

$$
\begin{aligned}
p_{2}(0)= & \widetilde{M}(0)+\left\|u_{0, x}\right\|_{L^{\infty}(S)} \\
& +\sqrt{\frac{\lambda^{2}}{\sigma^{2}}-\frac{K_{2}^{2}(T)}{\sigma}}-\frac{\lambda}{\sigma} \geq \widetilde{M}(0)+\left\|u_{0, x}\right\|_{L^{\infty}(S)} \geq 0 .
\end{aligned}
$$

We now claim that $p_{2}(t) \geq 0$, for any $t \in[0, T)$. Define

Suppose not, then there is $\widetilde{t} \in[0, T)$ such that $p_{2}\left(\widetilde{t_{0}}\right)<0$.

$$
t_{2}=\max \left\{t<\widetilde{t_{0}}: p_{2}(t)=0\right\} .
$$

Then, $p_{2}\left(t_{2}\right)=0$ and $p_{2}^{\prime}\left(t_{2}\right)<0$, or equivalently,

$$
\widetilde{M}\left(t_{2}\right)=-\left\|u_{0, x}\right\|_{L^{\infty}(S)}-\sqrt{\frac{\lambda^{2}}{\sigma^{2}}-\frac{K_{2}^{2}(T)}{\sigma}}+\frac{\lambda}{\sigma}
$$

and $\widetilde{M}^{\prime}\left(t_{2}\right) \leq 0$ a.e. $t \in[0, T)$. However, we have

$$
\begin{aligned}
\widetilde{M}^{\prime}\left(t_{2}\right)= & -\frac{\sigma}{2} \widetilde{M}^{2}\left(t_{2}\right)+\lambda \widetilde{M}\left(t_{2}\right)+\frac{1}{2} \gamma^{2}\left(t_{2}\right) \\
& +f\left(t_{2}, \varphi\left(t_{2}, x_{0}\right)\right) \quad \text { a.e. } t \in[0, T) \\
\geq & -\frac{\sigma}{2}\left(-\left\|u_{0, x}\right\|_{L^{\infty}(S)}-\sqrt{\left.\frac{\lambda^{2}}{\sigma^{2}}-\frac{K_{2}^{2}(T)}{\sigma}\right)^{2}}\right. \\
& +\frac{\lambda^{2}}{2 \sigma}-\frac{1}{2} K_{2}^{2}(T)>0 .
\end{aligned}
$$


Therefore, $p_{2}(t) \geq 0$ for any $t \in[0, T)$. Since $x$ is chosen arbitrarily, we obtain (46).

Let $\sigma=0$. Using previous arguments, (56) becomes

$$
\begin{gathered}
M^{\prime}(t)=-\frac{\sigma}{2} M^{2}(t)+\lambda M(t)+\frac{1}{2} \gamma^{2}(t)+f\left(t, \varphi\left(t, x_{0}\right)\right) \\
\gamma^{\prime(t)}=-\gamma M, \quad \text { a.e. } t \in[0, \mathrm{~T}),
\end{gathered}
$$

where the notation denotes the derivative with respect to $t$ and $f$ represents the function

$$
\begin{aligned}
f & =-A u-\lambda \partial_{x}^{-1} u+g(t) \\
& =-A u-\lambda \partial_{x}^{-1} u-\int_{S}\left(\frac{1}{2} \rho^{2}-A u\right) d x .
\end{aligned}
$$

We first compute the upper and lower bounds for $f$ for later use in getting the blow-up result:

$$
\begin{aligned}
f= & -A u-\lambda \int_{0}^{x} u d x-\frac{1}{2} \int_{S} \rho^{2} d x+A \int_{S} u d x \\
\leq & -A u-\lambda \int_{0}^{x} u d x+A \int_{S} u d x \leq \frac{A}{2}\left(1+u^{2}\right) \\
& +\frac{A-\lambda}{2}\left(1+\int_{S} u^{2} d x\right) \\
\leq & \frac{A}{2}\left(1+\frac{1}{2} \int_{S}\left(u^{2}+u_{x}^{2}\right) d x\right)+\frac{A-\lambda}{2}\left(1+\int_{S} u^{2} d x\right) \\
\leq & A-\frac{\lambda}{2}+\frac{A}{4} \int_{S}\left(\rho^{2}+u_{x}^{2}\right) d x+\frac{3 A-2 \lambda}{4} \int_{S} u^{2} d x \\
\leq & A-\frac{\lambda}{2}+\frac{A}{4} E_{0}+\frac{3 A-2 \lambda}{4}\left[e^{C_{2} t}\left(\int_{S} u_{0}^{2}(x) d x+1\right)\right] \\
\leq & A-\frac{\lambda}{2}+\frac{A}{4} E_{0}+\frac{3 A-2 \lambda}{4}\left[e^{C_{2} T}\left(\left\|u_{0}\right\|_{L^{2}(S)}^{2}+1\right)\right] .
\end{aligned}
$$

Now, we turn to the lower bound of $f$ :

$$
\begin{aligned}
-f & =A u+\lambda \int_{0}^{x} u d x+\frac{1}{2} \int_{S} \rho^{2} d x-A \int_{S} u d x \\
& \leq A \frac{1+u^{2}}{2}+\frac{1}{2} \int_{S} \rho^{2} d x+\frac{A-\lambda}{2}\left(1+\int_{S} u^{2} d x\right) \\
& \leq A-\frac{\lambda}{2}+\frac{A+2}{4} \int_{S}\left(\rho^{2}+u_{x}^{2}\right) d x+\frac{3 A-2 \lambda}{4} \int_{S} u^{2} d x \\
& \leq A-\frac{\lambda}{2}+\frac{A+2}{4} E_{0}+\frac{3 A-2 \lambda}{4}\left[e^{C_{2} T}\left(\left\|u_{0}\right\|_{L^{2}(S)}^{2}+1\right)\right] .
\end{aligned}
$$

Combining (82) and (83), we obtain

$$
|f| \leq A-\frac{\lambda}{2}+\frac{A+2}{4} E_{0}+\frac{3 A-2 \lambda}{4}\left[e^{C_{2} T}\left(\left\|u_{0}\right\|_{L^{2}(S)}^{2}+1\right)\right] .
$$

We know $M(t)>0$ for $t \in[0, T)$. From the second equation of (81), we obtain that

$$
\gamma(t)=\gamma(0) e^{-\int_{0}^{t} M(\tau) d \tau}
$$

Hence,

$$
\left|\rho\left(t, \varphi\left(t, x_{0}\right)\right)\right|=|\gamma(t)| \leq|\gamma(0)| .
$$

Therefore, we have

$$
\begin{aligned}
M^{\prime}(t) & =\lambda M(t)+\frac{1}{2} \gamma^{2}(t)+f\left(t, \varphi\left(t, x_{0}\right)\right) \\
& \leq \lambda M(0)+\frac{1}{2} \gamma^{2}(0)+\frac{1}{2} K_{1}^{2}(T), \\
M^{\prime}(t)-\lambda M(t) & \leq \frac{1}{2}\left(\sup _{x \in S} \rho_{0}^{2}(x)+K_{1}^{2}(T)\right) \quad \text { a.e. } t \in[0, T) .
\end{aligned}
$$

Integrating $(88)$ on $[0, t]$, we prove $(47)$ as follows:

$$
M(t) \leq \sup _{x \in S} u_{0, x}(x)+\frac{1}{2}\left(\sup _{x \in S} \rho_{0}^{2}(x)+K_{1}^{2}(T)\right) \frac{e^{\lambda t}-1}{\lambda} .
$$

To obtain a lower bound for $\inf _{x \in S} u_{x}(t, x)$, we use the same argument.

Since $\sigma=0$, (80) becomes

$$
\begin{gathered}
\widetilde{M}^{\prime}(t)=\lambda \widetilde{M}(t)+\frac{1}{2} \gamma^{2}(t)+f\left(t, \varphi\left(t, x_{1}\right)\right), \\
\widetilde{\gamma}^{\prime}(t)=-\widetilde{\gamma} \widetilde{M}, \quad \text { a.e. } t \in[0, T) .
\end{gathered}
$$

Because of $\widetilde{M}(t)<0$, we get from the second equation of (90) that

$$
\tilde{\gamma}(t)=\widetilde{\gamma}(0) e^{-\int_{0}^{t} M(\tau) d \tau}
$$

This means that

$$
\left|\rho\left(t, \varphi\left(t, x_{1}\right)\right)\right|=|\gamma(t)| \geq|\gamma(0)| .
$$

Then,

$$
\begin{aligned}
\widetilde{M}^{\prime}(t) & =\lambda \widetilde{M}(t)+\frac{1}{2} \gamma^{2}(t)+f\left(t, \varphi\left(t, x_{0}\right)\right) \\
& \geq \lambda \widetilde{M}(0)+\frac{1}{2} \gamma^{2}(0)+\frac{1}{2} K_{2}^{2}(T), \\
\widetilde{M}^{\prime}(t)-\lambda \widetilde{M}(t) & \geq \frac{1}{2}\left(\inf _{x \in S} \rho_{0}^{2}(x)-K_{2}^{2}(T)\right) \quad \text { a.e. } t \in[0, T) .
\end{aligned}
$$

Integrating (94) on $[0, t]$, we prove (48). This completes the proof of Lemma 14.

Lemma 15. Suppose that $\sigma \in R \backslash\{0\}$. Suppose $X_{0}=\left(\begin{array}{c}u_{0} \\ \rho_{0}\end{array}\right) \in$ $H^{s} \times H^{s-1}$ with $s \geq 2$, and let $T$ be the maximal existence time 
of the solution $X=\left(\begin{array}{l}u \\ \rho\end{array}\right)$ to (3) with initial data $X_{0}$. Then we have

$$
\rho(t, \varphi(t, x)) \varphi_{x}(t, x)=\rho_{0}(x), \quad(t, x) \in[0, T] \times S .
$$

Moreover, if there exists $M>0$ such that

$$
\inf _{(t, x) \in[0, T] \times S} \sigma u_{x}(t, x) \geq-M, \quad(t, x) \in[0, T] \times S .
$$

Then

$$
\begin{aligned}
\|\rho(t, \cdot)\|_{L^{\infty}(S)} & =\|\rho(t, \varphi(t, \cdot))\|_{L^{\infty}(S)} \\
& \leq e^{M T / \sigma}\left\|\rho_{0}\right\|_{L^{\infty}(S)} \quad(\sigma>0) \\
\|\rho(t, \cdot)\|_{L^{\infty}(S)} & =\|\rho(t, \varphi(t, \cdot))\|_{L^{\infty}(S)} \\
& \leq e^{N T / \sigma}\left\|\rho_{0}\right\|_{L^{\infty}(S)} \quad(\sigma<0),
\end{aligned}
$$

where $N=\left\|u_{0, x}\right\|_{L^{\infty}(S)}+\sqrt{\lambda^{2} / \sigma^{2}-K_{2}^{2}(T) / \sigma}-\lambda / \sigma$ and $K_{2}(T)$ is given in (50).

Proof. Differentiating the left hand side of (95) with respect to $t$, in view of the relations (12) and (3), we obtain

$$
\begin{aligned}
\frac{d}{d t}\{\rho & \left.(t, \varphi(t, x)) \varphi_{x}(t, x)\right\} \\
= & {\left[\rho_{t}(t, \varphi)+\rho_{x}(t, \varphi) \varphi_{t}(t, x)\right] } \\
& \times \varphi_{x}(t, x)+\rho(t, \varphi) \varphi_{x t}(t, x) \\
= & {\left[\rho_{t}(t, \varphi)+\rho_{x}(t, \varphi) \varphi_{t}(t, x)\right] \varphi_{x}(t, x) } \\
& +\rho(t, \varphi) u_{x}(t, \varphi) \varphi_{x}(t, x) \\
= & {\left[\rho_{t}(t, \varphi)+\rho_{x}(t, \varphi) \varphi_{t}(t, x)+\rho(t, \varphi) u_{x}(t, \varphi)\right] } \\
& \times \varphi_{x}(t, x)=0 .
\end{aligned}
$$

This completes the proof of (95). In view of the assumption (96) and $\sigma>0$, we obtain $u(t, x) \geq-(M / \sigma)(t, x) \epsilon$ $[0, T] \times S$.

By Lemma 3 and (95), we have

$$
\begin{aligned}
\|\rho(t, \cdot)\|_{L^{\infty}} & =\|\rho(t, \varphi(t, \cdot))\|_{L^{\infty}}=\left\|e^{-\int_{0}^{t} u_{x}(\tau, \cdot) d \tau} \rho_{0}(\cdot)\right\|_{L^{\infty}} \\
& \leq e^{M T / \sigma}\left\|\rho_{0}(\cdot)\right\|_{L^{\infty}} .
\end{aligned}
$$

To obtain (98), we use a similar argument as before. Using (13) and the lower bound for $u_{x}(t, x)$ in $(46)$, it follows that

$$
\begin{aligned}
\|\rho(t, \cdot)\|_{L^{\infty}} & =\|\rho(t, \varphi(t, \cdot))\|_{L^{\infty}}=\left\|e^{-\int_{0}^{t} u_{x}(\tau, \cdot) d \tau} \rho_{0}(\cdot)\right\|_{L^{\infty}} \\
& \leq e^{N T / \sigma}\left\|\rho_{0}(\cdot)\right\|_{L^{\infty}},
\end{aligned}
$$

which proves (98). This completes the proof of Lemma 15.
Proof of Theorem 12. Suppose that $T<\infty$ and that (42) is not valid. Then, there is some positive number $M>0$ such that

$$
\sigma u_{x}(t, x) \geq-M, \quad(t, x) \in[0, T] \times S .
$$

It now follows from Lemma 14 that $\left|u_{x}(t, x)\right| \leq C$, where $C=C\left(A, M, \sigma, E_{0}, \lambda,\left\|u_{0}\right\|, T\right)$. Therefore, Theorem 11 implies that the maximal existence time $T=\infty$, which contradicts with the assumption that $T<\infty$.

Conversely, the Sobolev embedding theorem $H^{s}(S) \rightarrow$ $L^{\infty}(S)$ with $s>1 / 2$ implies that if (70) holds, the corresponding solution blows up in finite time, which completes the proof of Theorem 12 .

\section{Acknowledgments}

The authors would like to thank the referee for comments and suggestions. This work is supported by the National Nature Science Foundation of China (no. 11171135), the Nature Science Foundation of the Jiangsu Higher Education Institutions of China (no. 09KJB110003), and the high-level talented person special subsidizes of Jiangsu University (no. 05JDG047).

\section{References}

[1] J. K. Hunter and R. Saxton, "Dynamics of director fields," SIAM Journal on Applied Mathematics, vol. 51, no. 6, pp. 1498-1521, 1991.

[2] Z. Yin, "On the structure of solutions to the periodic HunterSaxton equation," SIAM Journal on Mathematical Analysis, vol. 36, no. 1, pp. 272-283, 2004.

[3] H.-H. Dai and M. Pavlov, "Transformations for the CamassaHolm equation, its high-frequency limit and the Sinh-Gordon equation," Journal of the Physical Society of Japan, vol. 67, no. 11, pp. 3655-3657, 1998

[4] J. K. Hunter and Y. X. Zheng, "On a completely integrable nonlinear hyperbolic variational equation," Physica D, vol. 79, no. 2-4, pp. 361-386, 1994.

[5] R. Camassa and D. D. Holm, "An integrable shallow water equation with peaked solitons," Physical Review Letters, vol. 71, no. 11, pp. 1661-1664, 1993.

[6] R. S. Johnson, "Camassa-Holm, Korteweg-de Vries and related models for water waves," Journal of Fluid Mechanics, vol. 455, pp. 63-82, 2002.

[7] A. Constantin and B. Kolev, "On the geometric approach to the motion of inertial mechanical systems," Journal of Physics A, vol. 35, no. 32, pp. R51-R79, 2002.

[8] P. J. Olver and P. Rosenau, "Tri-Hamiltonian duality between solitons and solitary-wave solutions having compact support," Physical Review E, vol. 53, no. 2, pp. 1900-1906, 1996.

[9] R. Beals, D. H. Sattinger, and J. Szmigielski, "Inverse scattering solutions of the Hunter-Saxton equation," Applicable Analysis, vol. 78, no. 3-4, pp. 255-269, 2001.

[10] A. Bressan and A. Constantin, "Global solutions of the HunterSaxton equation," SIAM Journal on Mathematical Analysis, vol. 94, pp. 68-92, 2010.

[11] A. Bressan, H. Holden, and X. Raynaud, "Lipschitz metric for the Hunter-Saxton equation," Journal de Mathématiques Pures et Appliquées, vol. 94, no. 1, pp. 68-92, 2010. 
[12] J. K. Hunter and Y. X. Zheng, "On a nonlinear hyperbolic variational equation. I. Global existence of weak solutions," Archive for Rational Mechanics and Analysis, vol. 129, no. 4, pp. 305-353, 1995.

[13] J. Lenells, "The Hunter-Saxton equation describes the geodesic flow on a sphere," Journal of Geometry and Physics, vol. 57, no. 10, pp. 2049-2064, 2007.

[14] J. B. Li and Y. S. Li, "Bifurcations of travelling wave solutions for a two-component Camassa-Holm equation," Acta Mathematica Sinica (English Series), vol. 24, no. 8, pp. 1319-1330, 2008.

[15] W. Rui and Y. Long, "Integral bifurcation method together with a translation-dilation transformation for solving an integrable 2-component Camassa-Holm shallow water system," Journal of Applied Mathematics, vol. 2012, Article ID 736765, 21 pages, 2012.

[16] H. Wu and M. Wunsch, "Global existence for the generalized two-component Hunter-Saxton system," Journal of Mathematical Fluid Mechanics, vol. 14, no. 3, pp. 455-469, 2012.

[17] B. Moon and Y. Liu, "Wave breaking and global existence for the generalized periodic two-component Hunter-Saxton system," Journal of Differential Equations, vol. 253, no. 1, pp. 319-355, 2012.

[18] S. Wu and Z. Yin, "Blow-up, blow-up rate and decay of the solution of the weakly dissipative Camassa-Holm equation," Journal of Mathematical Physics, vol. 47, no. 1, Article ID 013504, 2006.

[19] S. Wu, J. Escher, and Z. Yin, "Global existence and blowup phenomena for a weakly dissipative Degasperis-Procesi equation," Discrete and Continuous Dynamical Systems B, vol. 12, no. 3, pp. 633-645, 2009.

[20] T. Kato, "Quasi-linear equations of evolution, with applications to partial differential equations," in Spectral Theory and Differential Equations, vol. 448 of Lecture Notes in Mathematics, pp. 25-70, Springer, Berlin, Germany, 1975.

[21] G. Gui and Y. Liu, "On the global existence and wave-breaking criteria for the two-component Camassa-Holm system," Journal of Functional Analysis, vol. 258, no. 12, pp. 4251-4278, 2010.

[22] A. Constantin and J. Escher, "Wave breaking for nonlinear nonlocal shallow water equations," Acta Mathematica, vol. 181, no. 2, pp. 229-243, 1998. 


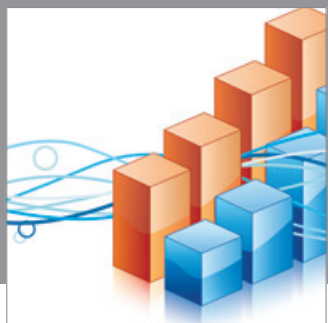

Advances in

Operations Research

mansans

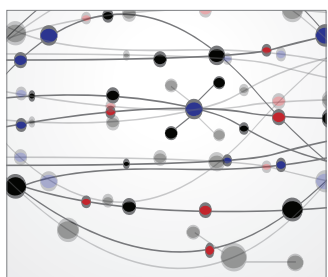

The Scientific World Journal
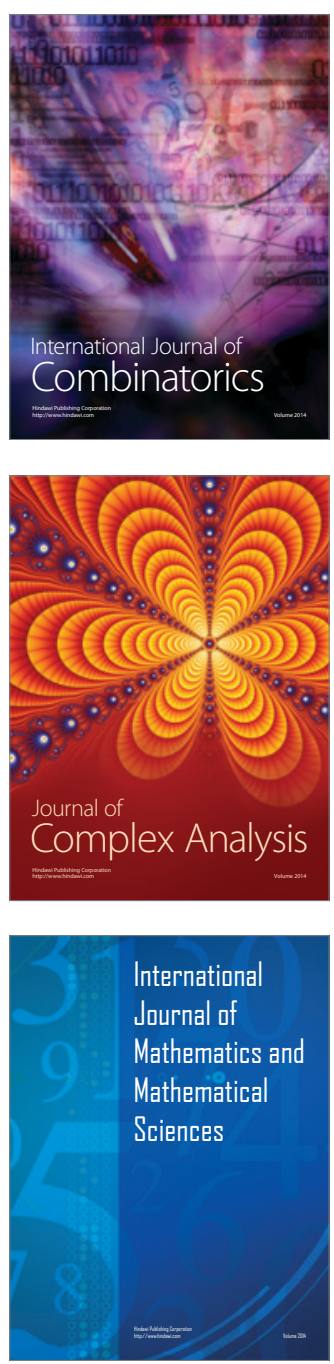
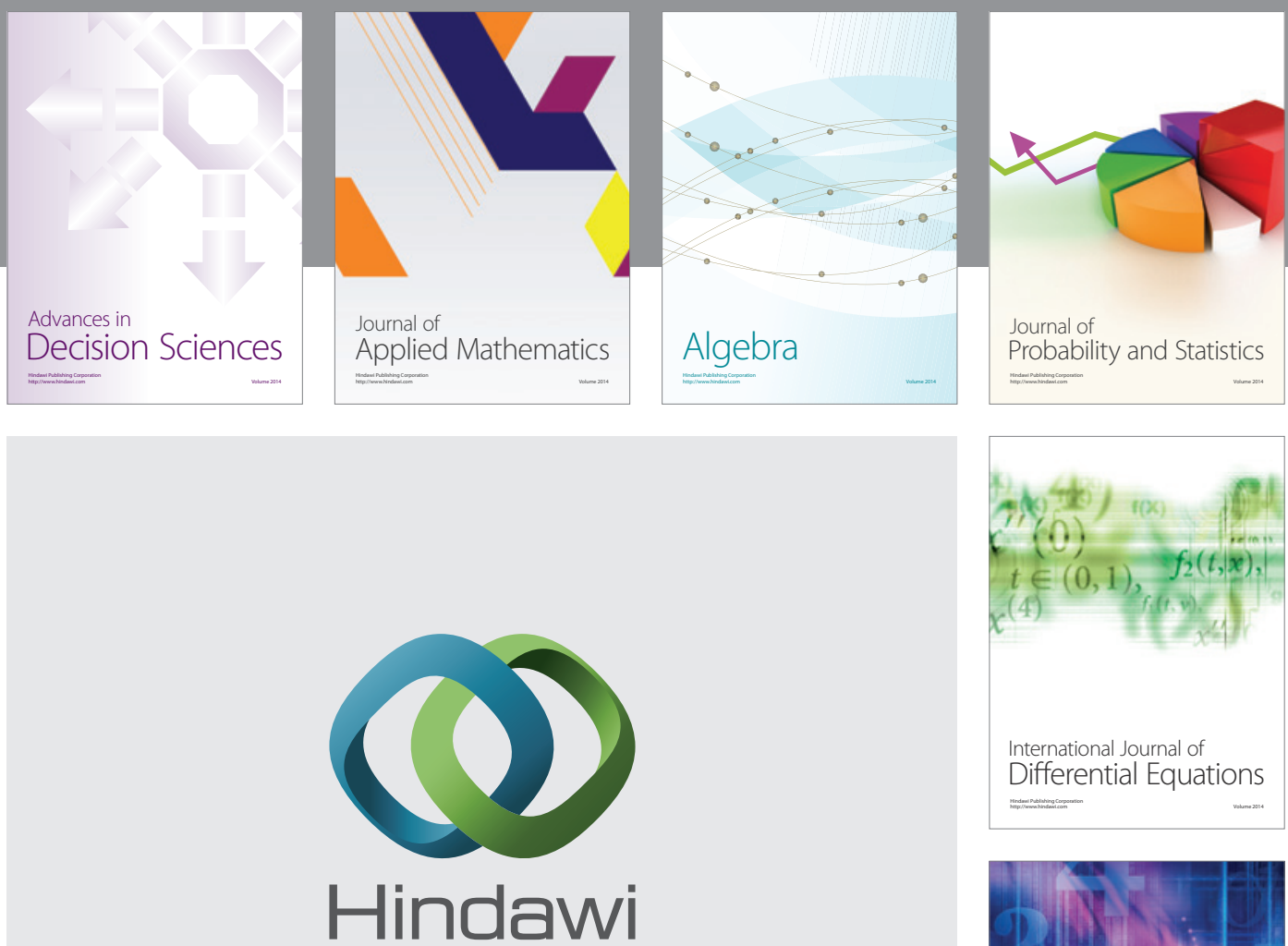

Submit your manuscripts at http://www.hindawi.com
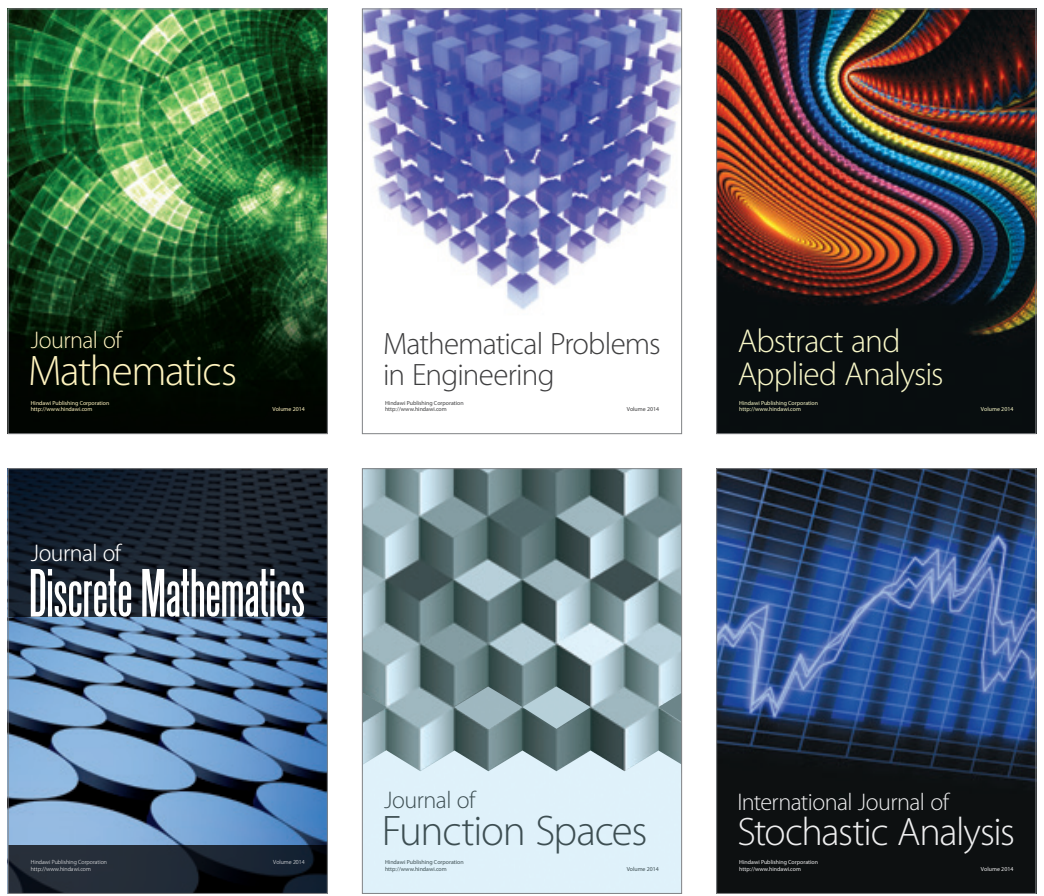

Journal of

Function Spaces

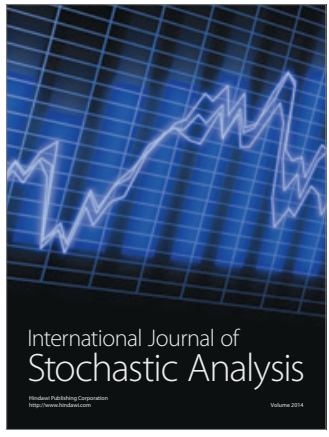

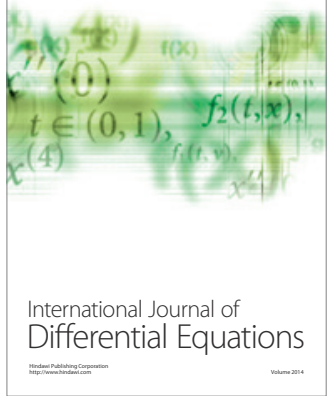
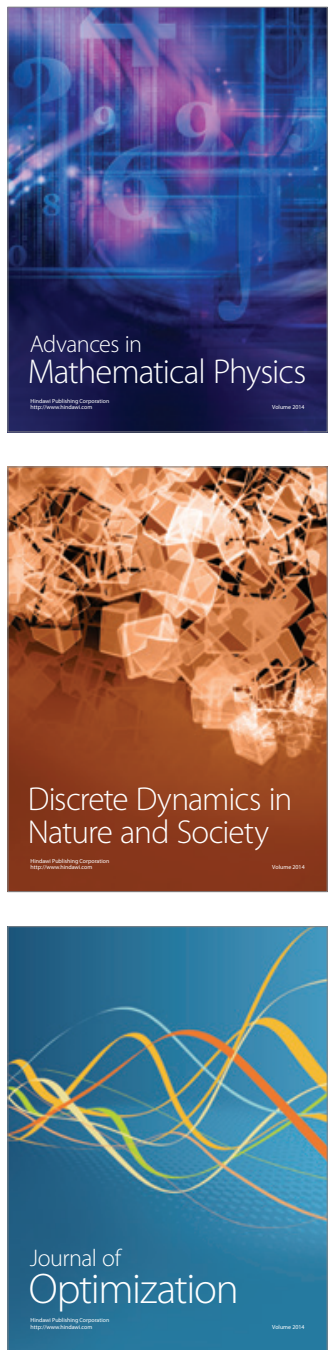